\title{
Comunicación en estrategias de mercadeo social del condón masculino para la prevención del VIH/sida en Cuba
}

\footnotetext{
Palabras clave

comunicación

mercadeo

condón masculino

estrategia

prevención

$\mathrm{VIH} /$ sida

\begin{abstract}
Resumen
Las estrategias de mercadeo social del condón masculino constituyen el eje central de la prevención y control ante la epidemia del VIH/sida y dentro de ellas, la comunicación ha jugado un papel fundamental al acompañar la disponibilidad de condones de calidad, en cantidades que cubran las necesidades y satisfagan la demanda de la población. Resulta interesante compartir la experiencia y los aprendizajes de lo acontecido en Cuba, por sus características muy peculiares al ser, en dicho contexto, un acontecimiento inédito.

La comunicación transita por diferentes momentos desde los primeros antecedentes en la década del 80 del pasado siglo con el inicio de la epidemia: el silencio, susurrando mensajes; las primeras voces, el inicio en la estrategia de mercadeo social, el tránsito hacia el posicionamiento de mercadeo y comunicación y, con ellos, del empleo del condón masculino para propiciar prácticas sexuales protegidas. Finalmente, concluyendo el primer decenio del siglo XXI, se produce el diálogo entre todos los actores: personal de salud, líderes de opinión, maestros, adolescentes, jóvenes, mujeres, hombres, HSH, grupos vulnerables y población en general.

Obstáculos y dificultades, desconocimiento, mitos, creencias y estigmas se van entrelazando en ese proceso, donde se demuestra el papel de la comunicación, en todas sus manifestaciones, cuando se diseña e implementa de forma adecuada, ya que propicia los cambios de comportamiento que se requieren para alcanzar el bienestar.
\end{abstract}

\author{
Nery Suárez Lugo \\ Escuela Nacional de Salud Pública \\ (La Habana, Cuba)
}

La autora declara no tener conflictos de interés. Nery Suárez Lugo forma parte del equipo editorial de Revista de Comunicación y Salud y no ha participado en el proceso de edición de este artículo. 


\title{
Male condom social marketing communication strategies for the prevention of HIVIAIDS in Cuba
}

\begin{tabular}{c}
\hline Keywords \\
\hline communication \\
social marketing \\
male condom \\
strategy \\
prevention \\
HIV/AIDS
\end{tabular}

\begin{abstract}
Male condom social marketing strategies are essential to the prevention and control of the HIV/ AIDS epidemic. Within them, communication has played a central role in accompanying the availability of quality condoms, in numbers which cover the needs and satisfy the demands of the population. The experience and lessons learned in Cuba are of interest due to the peculiar and unprecedented characteristics of these events as well as to their context.
\end{abstract}

During the 80's, communications went through different periods starting at the beginning of the epidemic: silence, whispered messages, the first voices, the start of a social marketing strategy, a transition toward communications and marketing positioning and with these the use of the male condom to encourage safe sexual relationships.

Finally, at the end of the first decade of the 21st century, a dialogue among all actors takes place: healthcare professionals, opinion leaders, teachers, adolescents, young adults, women, men, MSM, vulnerable groups and general population.

Obstacles and difficulties, lack of knowledge, myths, beliefs and stigmas are interlaced in this process where the role of communication is proven in all of its forms when adequately designed and implemented, as it promotes behavioral changes which are required in achieving wellbeing.

\section{Cómo citar el artículo}

Suárez Lugo, N. (2014). Comunicación en estrategias de mercadeo social del condón masculino para la prevención del VIH/sida en Cuba. Revista de Comunicación y Salud, Vol. 4, pp. 69-83.

DOI: http://doi.org/10.35669/revistadecomunicacionysalud.2014.4.69-83 


\section{Introducción}

Transcurrida más de una década del siglo XXI, a pesar de los esfuerzos que se realizan para prevenirlo, el $\mathrm{VIH} /$ sida sigue constituyendo una pandemia y su transmisión no presenta grandes diferencias geográficas, de sexo, escolaridad, raza o situación social y económica. (UNAIDS, 2012).

Todo parece indicar que la velocidad de propagación es mucho mayor que la de las acciones preventivas, por lo que compartir éxitos, fracasos y lecciones aprendidas, en gran medida puede ayudar a revertir la tendencia y detener la pandemia. La comunicación entre los que participan en este esfuerzo constituye también una tarea tan prioritaria como lo es comunicar a la población y a los grupos vulnerables las formas de prevenirla y actuar consecuentemente con una conducta responsable.

Es importante compartir las experiencias en lo referente al mercadeo social para posicionar el uso del condón masculino en la promoción de prácticas sexuales protegidas, ya que ha sido el eje central de las estrategias preventivas y de control ante la epidemia del VIH/sida. En ese marco, la comunicación ha jugado un papel fundamental al acompañar la disponibilidad de condones de calidad, en cantidades que cubran las necesidades y satisfagan la demanda de la población. (ONUSIDA, 2000).

La mercadotecnia social, también denominada como mercadeo, es una herramienta con una amplia gama de aplicación en la actividad sanitaria. En ese sentido, no se limita a la promoción de salud y el fomento de estilos de vida saludables sino que abarca también la calidad de los servicios de salud, el consumo racional de productos y servicios (en particular de los medicamentos) y contribuye a solucionar un amplio rango de problemas sociales y de salud pública que se generan.

En su dimensión social, la mercadotecnia social ofrece un campo nuevo de análisis e intervención, que se orienta a promover la aceptación y adopción de ideas, actitudes y prácticas en las personas para influenciarlas a aceptar, rechazar, modificar o abandonar conductas que ponen en riesgo su salud, calidad de vida y seguridad, generando cambios en diversos comportamientos sociales que propicien el bienestar del individuo, los grupos y la sociedad.

El diseño de una estrategia de mercadeo social se inicia con la investigación sobre los públicos metas. Además de identificar la prevalencia de un determinado comportamiento, también hay que saber acerca de los individuos, los grupos y el contexto. Uniendo los estudios cuantitativos y cualitativos, se realiza un análisis integral para encontrar las propuestas, con un panorama realista, previendo que los destinatarios tengan acceso económico para acceder a los productos y servicios que se quieren posicionar asociados a los comportamientos propuestos; considerando posibles desigualdades sociales; y derribando barreras sociales y psicológicas cuando resulte necesario, como el caso que nos ocupa del condón.

Para lograr el éxito, además de accesibilidad el mercadeo social requiere una estrategia de comunicación que permita difundir mensajes con información precisa, veraz 
y que no conduzca al error en relación a lo que se dice acerca de la idea, práctica y/o producto a promover; garantizar que los públicos metas tengan acceso al canal por donde se ofrece la información; y velar por el prestigio de la fuente y del informante, entre los aspectos de mayor relevancia. (Suárez, 2013).

Al referirse al mercadeo social del condón, la estrategia debe incluir la comunicación para informar sobre el producto, su disponibilidad y las ventajas de una relación sexual protegida, así como generar habilidades para enfrentar las barreras sociales y psicológicas, que aunque el condón esté disponible, pueden limitar su adquisición y uso. Ningún componente aislado puede lograr el efecto deseado, mientras que empleados en una adecuada combinación, conocida como mezcla de mercadotecnia, se potencian y se logra, aparejado al cumplimiento de los propósitos planteados, el máximo beneficio de los recursos empleados. (Suárez, 2013).

El rol de la comunicación es, por tanto, vital en toda estrategia de mercadeo social. Por sus características muy peculiares, resulta interesante de compartir la experiencia de la implementación para posicionar prácticas sexuales protegidas con el empleo del condón masculino en el escenario cubano.

\section{Creencias, actitudes y barreras}

En Cuba como en otros países, la información, la educación y la comunicación eran consideradas las únicas herramientas a utilizar para propiciar cambios de comportamientos en salud, a pesar del poco efecto demostrado (Suárez, 2004). Sin embargo, su inclusión como componente del mercadeo social no había constituido una práctica reconocida.

Son las experiencias empleadas para propiciar prácticas sexuales protegidas, como parte de los programas y proyectos de prevención del VIH/sida, los que amplían esa mirada de la salud pública internacional y en particular la introduce en Cuba. (Suárez, 2007).

Un problema de tal magnitud y la búsqueda de su solución fue más poderoso para la aceptación de la mercadotecnia social como estrategia de cambio de comportamientos en salud, que los múltiples esfuerzos realizados por especialistas del tema hasta esa fecha. La primera barrera social a romper, mediante una comunicación efectiva, fue dirigida a los decisores de las políticas públicas, que consideraban la mercadotecnia social como algo similar a la publicidad y solo posible de emplear con fines comerciales. Se impulsó una nueva perspectiva que permitiera abordar tan importante problema de salud desde la perspectiva social de la mercadotecnia social, con un enfoque no lucrativo y poblacional, cuando existían actitudes de rechazo y no aceptación (Suárez, 2008).

El otro gran reto de la comunicación estaba centrado en lograr llegar a una población que tenía arraigados mitos y estigmas con relación al uso del condón (Brito, García, Iraizoz, Jiménez, 2005). Por ello, en primer lugar había que derribar barreras psicológicas (García, Ledón, 2009) que, por encontrarse también en los dirigentes de los 
medios de comunicación social, impidieron en un primer momento emplearlos y luego llevaron a hacerlo de forma muy discreta.

De lo antedicho se hace evidente que dos elementos de vital importancia se entrelazan al analizar el contexto social en que se desarrolla la estrategia cubana. El primero está relacionado con la presencia de voluntad política para priorizar la salud de la población, pero que no se comprendiera la necesidad de emplear el mercadeo social en aquellos aspectos que tenían en su base la necesidad de cambios de comportamiento. La segunda, que los estigmas y mitos relacionados con el manejo público de la sexualidad no sólo se encontraban en la población, sino que se ponían de manifiesto en decisores de las políticas públicas, los encargados de la comercialización y los medios de comunicación social. (Suárez, 2007).

Si bien en otros países los condones masculinos se expenden en todo tipo de establecimiento comercial, una característica particular en el contexto cubano de aquel momento lo constituyó el hecho de que solo se vendían en farmacias. Esto conllevaba que las personas, de todas las edades, se sintieran cohibidas para adquirir los mismos en un mostrador, solicitándolo a un dependiente y rodeado de otros compradores que en general adquirían algún medicamento y muchas veces convivían en su propia comunidad.

Eso hacía que los interesados en comprar el condón encontraran también barreras sociales y psicológicas en el lugar de compra: adolescentes y jóvenes, por temor a que sus padres lo conocieran; los adultos casados, porque pudiesen pensar que eran infieles. Los vendedores de las farmacias no hacían visible el producto y también lo despachaban con la timidez que se derivaba del ya referido estigma que caracterizaba el manejo público relacionado con las relaciones sexuales y el uso del condón masculino como medio de protección (ENSAP, 2013).

A lo anterior se unía el hecho de que no se permitía promocionar el uso del condón masculino en los medios de comunicación social ni tampoco se aceptaba que se hiciera en el punto de venta, por lo que nada se podía decir acerca de él en la prevención del $\mathrm{VIH} /$ sida y la protección en las relaciones sexuales quedaba sujeta a la espontaneidad.

Lograr la accesibilidad requería que el lugar de venta fuera diferente. Aunque las máquinas automáticas no estaban disponibles, la creación de nuevos espacios para la comercialización, ubicados en bares, cafeterías, discotecas, centros nocturnos y de recreación, harían que los interesados se sintieran menos expuestos a una situación social y psicológica no favorecedora a la privacidad al realizar la compra.

A grandes rasgos, así es cómo se recibieron en Cuba al condón masculino, las estrategias de mercadeo social y la comunicación que tenían el propósito de posicionar prácticas sexuales protegidas ante la inminente situación de la epidemia del VIH/sida. (PNUD Cuba, 2012). 


\section{El silencio}

La ausencia de información y un vacío en la comunicación acompañaron los primeros acontecimientos relacionados con la epidemia del VIH/sida en la década del 80 del pasado siglo, aunque en el año 1983 se dictaron por parte del Ministerio de Salud Pública (MINSAP) las primeras medidas encaminadas a evitar la propagación en el territorio nacional.

En el año 1986 entró en vigor el "Programa de Control y Prevención del VIH/sida", centrado fundamentalmente en el diagnóstico y tratamiento. La comunicación se presentaba como parte de la educación para la salud, concebida para actuar de forma directa con la población a través del Sistema Nacional de Salud (SNS).

No se realizaba ninguna labor comunicacional para la promoción de relaciones sexuales protegidas ni entre los diagnosticados, siendo la abstinencia la medida a adoptar. Mientras tanto, se ofertaba una sola marca de condones, TWIN LOTUS, de fabricación china, en cantidad limitada y baja calidad, tamaño pequeño, y con un diseño poco atractivo, no acorde con la cultura de los cubanos, que tampoco contribuía a su uso.

Como contradicciones que afloran en el análisis de ese momento, cabe señalar que mientras se ofrecía total acceso a medios diagnósticos del VIH/sida y al tratamiento, no se disponía de condones masculinos de calidad, lo que se encuentra en correspondencia con la poca cultura existente sobre el tema y el predominio del enfoque curativo y asistencial sobre el preventivo.

Otra contradicción, más relacionada con las creencias y actitudes, es el hecho que el aborto fuera permitido y legal, (Herrera, Rodríguez, Quintero, 1997). Aunque se conoce ya en ese momento que las relaciones sexuales no protegidas son la fundamental vía de contagio, los cubanos no acostumbraban a usar el condón masculino y se mantenían los estigmas asociados al manejo de la comunicación sobre el uso del condón como estrategia preventiva.

Este momento se caracterizaba por no hablar del tema, tanto en los medios de comunicación social como en los espacios de que disponía el SNS. La ausencia de la comunicación es también el reflejo de que las creencias y actitudes de los encargados de llevar a vías de hecho el Programa, que evitaban el manejo público de lo relacionado con las relaciones sexuales a pesar de las evidencias al respecto (PNUD, 2013).

\section{Susurrando mensajes}

Se inicia el trabajo asistencial a cargo ya del MINSAP con los diagnosticados con el $\mathrm{VIH} /$ sida, que se ejecuta con pasos certeros para ofrecer atención, apoyo y prevención en los Centros Asistenciales a través de los grupos denominados GPsida (Aragonés, Campos, Sánchez, Pérez, 2007). Como parte de la atención a los diagnosticados, se inicia la orientación de forma directa para prevenir el contagio en las relaciones sexuales. 
Los esfuerzos preventivos continúan con programas tradicionales de educación para la salud. Poco se avanza en los primeros años de la década del 90 con relación a la comunicación, ya que la información que se difunde se centra en la estabilidad, la fidelidad, la selectividad de la pareja y la responsabilidad individual.

No se habla del condón en los medios de comunicación social. Solo alguna información de manera discreta, la producción y distribución del primer tabloide educativo y algunas actividades de adiestramiento. Se producen los primeros carteles y el primer material impreso, denominado "Nosotros", de orientación a los seropositivos, que por ser valorado como inadecuado, fue poco difundido.

Prevalecen los estigmas y, por tanto, era muy escasa la comunicación acerca de las relaciones sexuales protegidas, aunque resultaría de gran utilidad para desmitificar el rechazo al condón masculino asociado a la disminución del placer. También se mantiene la percepción de mala calidad, que se sustenta en la presencia de los condones que se ofertaban y por tanto no facilitan la promoción de su uso.

La orientación a la población sexualmente activa, y dentro de ella a los grupos más vulnerables, se encuentra todavía ausente. (PNUD, 2012). La comunicación no acompaña la presencia de la epidemia del VIH/sida que ya se instaura en el escenario cubano.

\section{Las primeras voces}

La segunda mitad de la década del 90 se inicia con el apoyo al MINSAP de la Organización No Gubernamental "Médicos sin Frontera" de Holanda, con un proyecto para prevenir Infecciones de Transmisión Sexual, promoviendo relaciones sexuales protegidas mediante el uso del condón masculino y donando al país condones más atractivos y de mejor calidad.

Una mirada diferente, proveniente de países con mayor desarrollo y donde la sexualidad no constituye un tema tabú, hacen que la comunicación y mayor disponibilidad se den por primera vez la mano. Se pone en práctica el "Plan Intersectorial para la Educación, Prevención y Control de las ITS/VIH/sida" y el "Programa de Prevención y Control de Enfermedades de Transmisión Sexual", que tenía como objetivo desarrollar el mercadeo social de condones con la participación de los sectores y organismos afines. (Rojo, Pérez, Llanusa, Álvarez, Suárez, 2008).

Sin embargo, la comunicación no llega a los medios. Solo queda incluida en un Componente de Información, Educación, Comunicación, IEC, para fomentar una disposición favorable al uso del condón, con la necesidad de que la disponibilidad se acompañe del entrenamiento a las personas para su uso correcto, así como al personal de salud para poder lograr tal propósito, haciendo que esas primeras voces solo se escuchen dentro de las diferentes instancias del SNS (Herrera, Rodríguez, Quintero, 1997).

Organismos y organizaciones internacionales con experiencia en el manejo de estrategias preventivas contribuyen en la segunda mitad de la década del 90 a desarrollar acciones y proyectos de colaboración. En 1998, el MINSAP crea el Centro Nacional de 
Prevención de las ITS-VIH/sida, para coordinar todo el trabajo en esta dirección como parte de un proyecto de colaboración con ONU/sida. (Centro Nacional de Prevención ITS-VIH/sida, 2006).

Pero quienes deciden las políticas públicas mantienen los estigmas asociados al manejo público del uso del condón con el empleo del mercadeo social. No se permite emplear los medios de comunicación masiva para emitir mensajes sobre el tema; aunque se dispone de programa dedicados a la salud y en el trabajo educativo con la población en el SNS, este sólo se visualiza de manera discreta. (PNUD, 2013).

También se mantienen los tabúes en los vendedores de las farmacias, que no hacen promoción ni visible el condón, y en la población, que no se atreve a comprarlo en esos establecimientos por el temor a ser enjuiciados por las personas que allí se encuentren y también el rechazo a su uso.

Si bien todo lo anterior apunta a la imperiosa necesidad de una adecuada estrategia de comunicación, que maneje de manera integral la necesaria modificación de creencias, actitudes y valores, tanto de los actores como de la población, no fue posible en ese momento implementarse, quedando un buen camino por recorrer hasta poder lograrlo.

\section{La comunicación comienza con el nuevo siglo}

Comenzando el siglo XXI se pone en marcha el "Plan Estratégico Nacional ITS/VIH/ sida. 2001-2006". Se aprueba un proyecto de colaboración entre la agencia especializada Population Service International, PSI y el MINSAP, centrado en el mercadeo social para promover prácticas sexuales protegidas mediante el uso del condón masculino. Disponibilidad y una campaña publicitaria son los ejes fundamentales de la estrategia basada en una investigación de mercados, que permitió identificar marca, precio, lugares de venta y grupos diana, en las edades entre 15 y 39 años de áreas urbanas, con mayor incidencia en ITS- VIH/sida (MINSAP, 2006).

Para facilitar la accesibilidad, se crean los Puntos de Venta No Tradicionales (PVNT), seleccionados por ser lugares donde se realizan encuentros que propician las relaciones sexuales de riesgo. Además, la población que acude a los PVNT no tiene la misma composición social que en las farmacias, que se encuentran situadas en la comunidad y a la que concurren fundamentalmente mujeres amas de casa y adultos mayores (Suárez, Rodríguez, 2006).

La razón de ser de los PVNT fue acercar el producto al consumidor como base de la accesibilidad y que este lugar tuviese condiciones diferentes a los que ya existían para ser favorecedores a la adquisición del producto, tanto por los lugares donde se encontraban ubicados. Por ello, en la estrategia de mercadeo se les denominó como Puntos de Venta No Tradicionales, mientras que las farmacias comenzaron a llamarse Puntos de Venta Tradicionales (PVT).

La comunicación también tuvo un nuevo enfoque, ya que comienzan a difundirse mensajes sobre el uso del condón masculino con un lema y mediante carteles, medios 
impresos y una canción, pero con dificultad por la presencia de opositores.

Prevalecen los insuficientes conocimientos en estrategias de mercadeo social y el desconocimiento del manejo logístico del condón para garantizar su accesibilidad. Pero, a la vez, se van haciendo visibles las diferencias entre las estrategias de mercadeo social y las educativas tradicionales, apuntando, aun discretamente, a la efectividad de las primeras.

Aunque el país contaba con especialistas de elevada calificación, dada la situación socio- económica y de mercado no habían enfrentado una tarea de esta naturaleza. Si bien comienzan a apropiarse de los conocimientos para el manejo del mercadeo social, aun no se habían desarrollado las habilidades requeridas que solo la experiencia proporciona, mucho más cuando se trata del contexto cubano que carecía de antecedentes al respecto.

Generar una demanda insatisfecha de condones masculinos en aquellos territorios a los que no llegaba la cobertura del proyecto, pero a donde si la comunicación hacía saber sobre el tema, constituye una dificultad y un propósito. La intención de compra sin respuesta disminuye el efecto deseado con la comunicación, al provocar insatisfacción en los consumidores potenciales (Suárez, 2007).

\section{Mercadeo y comunicación hacia el posicionamiento}

A comienzos del siglo XXI, la experiencia del mercadeo social y sus componentes, donde la comunicación juega un papel fundamental, resultan inéditos en el contexto cubano. Por vez primera se diseña e implementa un proyecto de mercadeo social para promover conductas saludables; se aborda desde el espacio público el tema de las relaciones sexuales; se ofertan condones en otros lugares que no sean las farmacias; y se promociona su uso en los puntos de venta y en los medios de comunicación social. El posicionamiento de la mercadotecnia social constituye el primer eslabón para lograr el uso del condón en la población cubana.

La cooperación internacional sigue avanzando. La unión del MINSAP con PSI y el Fondo Mundial de lucha contra el Sida, la malaria y la tuberculosis (FM) hace que el "Programa Nacional de Prevención y Control del VIH/sida" y el "Plan Estratégico Nacional para ITS/VIH/sida 2001-2006", (MINSAP, 2006) se integren en la ejecución de proyectos que reciben apoyo financiero y material a través del FM. Este proceso comienza con una investigación de mercado para lanzar una marca cubana de condones, que ofrecerá la información requerida para segmentar la población y dirigir la estrategia de comunicación a los grupos meta prioritarios, tomando como referente la información epidemiológica del comportamiento de la epidemia (PNUD/FM/cub, 2003).

Los proyectos con el FM se mantienen hasta el año 2012 (PNUD/FM/cub, 2007) (PNUD/FM/cub, 2008), y se logra una mayor cobertura a nivel nacional para la distribución en el mercado y por el SNS de muestras gratis de condones de color, sabor y texturizados. La continuidad se produce también gracias a los esfuerzos locales para hacerlo sostenible. Se dispone al finalizar la primera década del siglo XXI de la marca 
cubana de condones, VIGOR; también se ofertan lubricantes con la marca VIGOR MAX y se extiende la venta a máquinas automáticas.

La comunicación por los medios masivos se va ampliando. Se emiten mensajes dirigidos a la vulnerabilidad social, fundamentalmente al grupo más afectado, los hombres que tienen sexo con hombres (HSH), y a los hombres en general, en función de la competitividad y audacia, así como otros dirigidos a los jóvenes y a las mujeres. Los grupos metas van variando en función de la situación epidemiológica del VIH/sida a nivel nacional y por territorios.

La enseñanza de cómo usar el condón masculino de forma correcta y la negociación en la pareja forman parte de la estrategia de comunicación, pero esto se realiza cara a cara, mediante los promotores o en talleres con los grupos de mayor vulnerabilidad.

Sin embargo, centrar la comunicación, como componente de la estrategia de mercadeo social, en el condón que tiene menor disponibilidad y accesibilidad en el mercado, tanto en cantidad como en puntos de venta y precio, lleva a una demanda insatisfecha de esa marca. Esta situación se agudiza en el año 2008 ante las dificultades en la importación y la ausencia del condón de mercadeo social, ya sea para la venta como para la distribución gratuita; una situación que, aunque fue resuelta en breve tiempo, condujo a una cierta incertidumbre en la población y a que la accesibilidad no estuviera en correspondencia con la información difundida.

Las ventas de condones en general, y en particular de los condones de mercadeo social VIVE, VIGOR y VIGOR MAX, el incremento en el uso y la aceptación manifestada por la población, hicieron visible el posicionamiento de la marca. Los condones que se distribuyen para la venta y entrega gratuita son de alta calidad avalada; no obstante, el almacenamiento inadecuado en los territorios y PVNT puede deteriorarlos y llegar al consumidor sin la calidad requerida, con las consecuencias negativas sobre la seguridad en la relación sexual y posterior falta de credibilidad en su efecto protector difundida por la comunicación. (Suárez, 2007).

En los diferentes momentos en que ha sido evaluado el componente de comunicación de la estrategia de mercadeo social, se ha encontrado que resulta pobre la percepción de riesgo con la pareja estable e insuficiente la capacidad de las mujeres jóvenes de negociar con sus parejas del uso del condón, especialmente en grupos vulnerables (jóvenes, hombres que tienen sexo con otros hombres y mujeres).

Un estudio realizado comparando los años 2001, 2005, 2006, 2009 y 2011, permitió constatar que se ha incrementado paulatinamente el uso del condón en las relaciones sexuales ocasionales entre las personas de 15 a 34 años. El mayor número de usuarios continúa concentrándose en las poblaciones más jóvenes. Mientras tres de cada cuatro personas $(70,8 \%)$ casadas-unidas o con pareja estable tuvieron relaciones sexuales desprotegidas, tres de cada cuatro personas con parejas ocasionales se protegieron.

Evolutivamente, el uso del condón se incrementó más en hombres que en mujeres: en varones subió del 40,85 en 2006 al 48,04\% en 2009; en mujeres, la subida fue 
menor, del $33,26 \%$ en 2006 al $37,63 \%$ en 2009 . Como se puede observar, las prácticas sexuales protegidas mediante el uso del condón no se producen aún en la magnitud requerida (Oficina Nacional de Estadísticas e Información, 2013).

Siguiendo la ruta de la aceptación del empleo del mercadeo social del condón masculino, a partir del año 2008 se ha consolidado con ella su aceptación por las autoridades competentes del SNS, la población, los medios de comunicación social y los sectores involucrados. Se puede considerar que se ha posicionado y la comunicación, al ser coherente con los restantes componentes de la estrategia, comenzó a jugar el rol que le corresponde. (PNUD, 2012).

\section{Comunicación para difundir herramientas}

En el proceso de gestión del mercadeo de condones se construyó una caja de herramientas, tanto metodológicas como instrumentales, que permiten su perfeccionamiento en otros momentos del desarrollo, así como su posible utilización, previo análisis contextual, en otros proyectos. Estas herramientas permiten generar conocimientos e intervenir para lograr el propósito del mercadeo social en la planeación, comunicación, logística, fortalecimiento de capacidades y en el diagnóstico, monitoreo y evaluación. Para lograrlo se requiere también de la comunicación.

Por tal razón, el Programa de Naciones Unidas en su oficina de Cuba, está produciendo la serie "Aprendiendo y Compartiendo" para difundir las lecciones aprendidas en el esfuerzo de controlar la epidemia del VIH/sida.

Herramientas metodológicas como proyectos, documentos, convenios, guías, manuales, metodologías, programas de talleres de capacitación y adiestramientos, están apegados al marco conceptual que sustenta el mercadeo de condones y dan respuesta a necesidades del desempeño. Su difusión, mediante una estrategia comunicacional que facilite estar al alcance de los vinculados a estas acciones, puede acortar la brecha existente, con menores esfuerzos, entre la situación actual y a la que se aspira (United Nations, 2012).

De gran importancia son las herramientas instrumentales, pues facilitan la aplicación práctica e incluyen el diseño de campañas acordes a los distintos momentos del proceso de desarrollo, transitando por las etapas que responden a los resultados de los estudios de monitoreo y evaluación realizados desde el diseño hasta la implementación de los proyectos. Para cada campaña se produjeron objetos promocionales, soportes educativos, así como documentos informativos y audiovisuales que pueden formar parte de otros proyectos similares, luego de un análisis contextual que lo permita.

Forman parte también de las herramientas instrumentales los cuestionarios empleados para el diagnóstico, monitoreo y evaluación, que puestos a disposición de los investigadores del tema y difundidos en revistas científicas especializadas, facilitan el proceso de compartir experiencias comunes y lograr que los resultados cada vez sean mejores. 
Identificar las herramientas fue producto de la sistematización de la experiencia del Mercadeo Social del condón masculino para posicionar prácticas sexuales protegidas en la prevención del VIH/sida en Cuba, en el periodo 2001 - 2010 realizado por la Unidad de Programa VIH/sida y TB de la oficina del PNUD en Cuba. La comunicación fluida que se produjo entre la especialista que realizara la sistematización, autora del presente trabajo, y los actores que participaron en esta experiencia constituyó un elemento de vital importancia, tanto para obtener la información como para compartirla posteriormente. La socialización permitió que la identificaran y se apropiaran de ella, lo que demuestra una vez más cómo la comunicación de doble sentido a la vez tributa a la sostenibilidad del proyecto, su enriquecimiento y su perfeccionamiento (PNUD, 2012).

\section{Del silencio al diálogo: aprendiendo de la experiencia}

La teoría define de forma muy clara cómo se debe implementar una estrategia, pero siempre queda como asignatura pendiente cómo se hace en la práctica concreta. Esa es la brecha más importante, para la cual la experiencia acumulada facilita las herramientas que se deben emplear para acortarlas, ya que todos los contextos, si bien en algunos aspectos resultan diferentes, en otros son similares.

Las lecciones aprendidas en esta experiencia del mercadeo social del condón masculino para el posicionamiento de prácticas sexuales protegidas en la prevención del VIH/ sida en Cuba y el papel que en ella ha jugado la comunicación pueden ser de utilidad para el empleo de otras estrategias con ese u otros propósitos.

Entre las lecciones más relevantes se puede señalar la importancia de hacer visible mediante su difusión, la utilidad en la prevención y control de la epidemia; compartir la información que se dispone acerca del contexto social y cultural; y las oportunidades, amenazas, fortalezas y debilidades que fueron identificadas, que sustentan las acciones desarrolladas.

Como informaciones de gran utilidad para el diseño y el desarrollo de la estrategia, se encuentran el disponer de un adecuado proceso de obtención de información epidemiológica estratificada, resultados de encuestas de indicadores de prevención, que sólo se logran cuando existe una buena comunicación entre las diferentes instancias de salud con los donantes y aquellos que tienen a su cargo los proyectos.

La comunicación también permitirá convertir en aliados otros proyectos de cooperación internacional y nacional para propiciar la mayor cobertura de exposición para alcanzar los grupos meta.

Es importante aprender a emplear todas las formas disponibles y adecuadas para emitir los mensajes que se requieren en cada momento de ejecución, para que se complementen unas y otras; utilizar el enfoque socio cultural en la elaboración; desarrollar estrategias con acciones directas para los grupos vulnerables, en riesgo y la población en general, para aumentar la percepción de riesgo en función de sus particularidades; centrar la comunicación en la importancia del uso del condón masculino en las prácticas sexuales y en el desarrollo de habilidades para negociarlo en la pareja; así como 
emplear mensajes que incorporen el elemento erótico y no se centren solo en la enfermedad.

Hay que lograr la credibilidad de la información apoyándose en la calidad del producto, las fuentes emisoras y los comunicadores seleccionados. Siempre que se disponga de los recursos, es recomendable utilizar los servicios de agencias publicitarias como asesores para la comunicación social dada su experticia en el tema.

El transitar por esta experiencia, con múltiples obstáculos en el camino, permitió llegar del silencio acerca de la epidemia y sus causas, al diálogo, tanto en la pareja, como entre maestros y estudiantes, salubristas y comunicadores, así como con los líderes de opinión, artistas y deportistas con la población, todos involucrados en las campañas a través de los medios de comunicación social.

La amplia cobertura de mensajes sobre el condón en los medios de comunicación social que hoy día se cuenta es el mejor ejemplo de que la comunicación no es solo un eje transversal en toda estrategia de mercadeo social y su hilo conductor, sino que además es el elemento central para lograr la comprensión de su necesidad, de que el elemento educativo por sí solo no tiene el mismo efecto y de que el contexto tiene que resultar favorecedor a los comportamientos de salud que queremos alcanzar.

\section{Referencias}

Aragonés, L.C. Campos, D. Sánchez, V.L. y Pérez Ávila, L.J. (2007). Grupos de Prevención del SIDA (GPSIDA): 15 años de trabajo sostenido en la prevención del VIH/ sida. Revista Cubana de Medicina Tropical. Vol. 59, nº 3.

Brito, S.G. García, R.X. Iraizoz, B. y Jiménez H. (2005). Conocimientos y creencias de una población cubana sobre el VIH/SIDA desde un enfoque bioético. La Habana. Revista Cubana de Medicina General Integral. Vol. 22, nº 4.

Centro Nacional de Prevención ITS-VIH/sida (2006). Documento de Planificación Estratégica. Línea de Mercadeo Social de Condones. La Habana: Centro Nacional de Prevención ITS-VIH/ sida.

Escuela Nacional de Salud Pública (ENSAP) (2013). Evaluación Independiente del Proyecto "Avance hacia la sostenibilidad de la respuesta nacional para la prevención y atención de la epidemia del VIH/sida en la República de Cuba" 2008-2012. La Habana: ENSAP.

García, C.T. y Ledón, L. (2009). Sexualidad y adolescencia: Construcciones de género y barreras para el uso del condón en Cuba. En: Álvarez L, Rodríguez A. (Eds). Salud sexual y reproductiva en adolescentes cubanos. La Habana: Editora Abril.

Herrera, G.V. Rodríguez, D.L. y Quintero S.M. (1997). Anticonceptivo en la consulta de planificación familiar. Revista Cubana de Medicina General Integral. Vol. 13, n 4, pp. 345-351. 
ONUSIDA (2000). Programa Nacional de Sida. Guía para el monitoreo y evaluación. Ginebra: ONUSIDA.

Ministerio de Salud Pública de Cuba (MINSAP) (2006). Plan estratégico Nacional ITS/ VIH/sida 2001-2006 República de Cuba. Resolución Ministerial 110. La Habana: MINSAP.

Oficina Nacional de Estadísticas e Información de Cuba (ONEI) (2013). Encuesta sobre Indicadores de Prevención de Infección por VIH-2011. La Habana: ONEI.

PNUD (2008). Fortalecimiento de la respuesta nacional multisectorial para la prevención y atención de la epidemia del VIH/sida en la República de Cuba. 2003-2008. La Habana: PNUD/FM/Cuba.

PNUD (2009). Expansión y descentralización de acciones, para la prevención del VIH/ SIDA y para la atención integral y apoyo a personas viviendo con VIH/ sida en la República de Cuba 2007-2009. La Habana: PNUD/FM/Cuba.

PNUD (2012). Avance hacia la sostenibilidad de la respuesta nacional para la prevención y atención de la epidemia del VIH/sida en la República de Cuba 2008-2012. La Habana: PNUD/FM/Cuba.

PNUD (2012). Mercadeo Social del condón masculino para posicionar prácticas sexuales seguras en la prevención del VIH/sida en Cuba 2001/2010. Serie Aprendiendo y compartiendo. La Habana: PNUD.

Rojo, P.N. Pérez, P.J. Llanusa, R.S. Álvarez, V.L. y Suárez, N. (2008). Evaluación Independiente del Proyecto "Fortalecimiento de la respuesta nacional multisectorial para la prevención y atención de la epidemia del VIH/sida en la República de Cuba" [CD-ROM]. La Habana: Escuela Nacional de Salud Pública.

Suárez, N. (2004). Marketing y salud: una perspectiva teórico-práctica. $3^{a}$ Edición. Cali: Editorial CATORSE.

Suárez, N. y Rodríguez, A. (2006). Conocimiento, actitud y uso del condón masculino en la población en riesgo de contraer VIH/SIDA. Revista Horizontes Sanitarios. Vol. 5, $\mathrm{n}^{\circ} 1$, pp. 12-22.

Suárez, N. (2007). Enfoque Social de la Mercadotecnia Sanitaria. La Habana: Editorial Ciencias Médicas.

Suárez, N. (2007). Evaluación de la estrategia de mercadotecnia social del condón en Cuba, año 2005. Revista Cubana de Salud Pública. Vol. 33, nº 1, pp. 1-9.

Suárez, N. (2008). Mercadotecnia en el Sistema Nacional de Salud de Cuba, año 2005. Revista Cubana de Salud Pública. Vol. 34, n 1, pp. 1-11. 
Suárez, N. Tamargo, B. y Forcelledo, L. (2009). Conocimientos, actitudes y uso del condón masculino en la prevención del VIH/sida en estudiantes de la Facultad de Ciencias Médicas. Pinar del Río, Curso 2006-2007. Revista Horizontes Sanitarios. Vol. 8, n 1, pp. 7-24.

Suárez, N. (2013). Conferencia magistral. Simposio Internacional Red Iberoamericana Mercadotecnia en Salud. Santiago de Chile.

Suárez, N. (2013). Mercadeo social como herramienta en función de la salud sexual en Cuba. Revista Cubana de Salud Pública. Vol. 39, Supl. 1.

UNAIDS (2012). Together we will end AIDS. Ginebra: UNAIDS/OMS.

United Nations (2012). The Millennium Development Goals. Report 2012. Nueva York; ONU. 